\title{
Pandemia y participación política de los jóvenes en América Latina ${ }^{1}$
}

\section{Pandemic and political participation of young people in Latin America}

\author{
Olivia Leyva Muñoz ${ }^{2}$
}

\section{Resumen}

En un contexto de crisis provocada por la pandemia, el objetivo de la investigación es presentar una reflexión sobre el interés de los jóvenes por participar en política durante los procesos electorales del 2021, específicamente en las elecciones presidenciales de Chile, Perú, Nicaragua y Honduras, así como las elecciones intermedias que se realizarán en México y Argentina, en un año donde la pobreza y la desigualdad aumentan significativamente por la crisis económica que enfrentan los países en América Latina.

Palabras clave: participación política; juventudes; democracia; América Latina

\begin{abstract}
In a context of crisis caused by the pandemic, the aim of the research is to present a reflection on the interest of young people to participate in politics during the 2021 electoral processes, precisely in the presidential elections in Chile, Peru, Nicaragua and Honduras. As well as the midterm elections that are going to be held in Mexico and Argentina. In a year where poverty and inequality employed has been significantly raised due to the economic crisis faced by countries in Latin America.
\end{abstract}

Keywords: political participation; youth; democracy; Latin America

\section{Introducción}

La pandemia actual, causada por el virus SARS-CoV-2, ha transformado la vida de todas las personas que habitamos en el planeta, cuyos efectos también son analizados desde la perspectiva de las ciencias sociales para realizar aportes significativos en la revalorización de los escena-

\footnotetext{
1 Trabajo recibido el 28/03/2021. Aceptado el 07/06/2021.

${ }^{2}$ Universidad Autónoma de Guerrero. Sistema Nacional de Investigadores del Consejo Nacional de Ciencia y Tecnología (México). Contacto: olivialeyva@uagro.mx
} 
rios socio políticos actuales que se entrelazan constantemente en las naciones latinoamericanas, derivado de los procesos electorales próximos, que se desarrollarán en un contexto de pandemia y crisis económica.

La dinámica social interrumpida súbitamente por la pandemia afecta de manera diferente a los actores políticos, así como a los procesos electorales cercanos, tal es el caso de los jóvenes, en quienes el distanciamiento social ha impactado sobre sus emociones, la ocupación laboral, la educación y, por ende, en las oportunidades de bienestar y desarrollo social que ya no serán iguales después de la pandemia, al menos no para los cerca de 160 millones de 15 a 29 años que habitan en la región latinoamericana (CEPAL, 2021), quienes han tenido que sortear la enorme brecha de desigualdad estructural que ha predominado en la región durante décadas.

En este contexto, el propósito de este trabajo es presentar un análisis sobre ¿cuál será el interés de los jóvenes por involucrarse políticamente en las elecciones presidenciales del 2021 en Chile, Perú, Nicaragua y Honduras, así como las elecciones intermedias que se celebrarán en Argentina y México, en un contexto de pandemia? La hipótesis que se plantea para este trabajo es que las condiciones económicas, derivadas de la pandemia, limitarán la participación de los jóvenes en los procesos electorales.

En principio, se revisó la estrategia para promover la participación política de los jóvenes en los sistemas presidencialistas de América Latina, así como las condiciones económicas y de vulnerabilidad que predominan en la región, específicamente en los países en cuestión. En seguida, se resumen los referentes teóricos sobre la participación política y los mecanismos a través de los cuales se manifiesta (Lazarsfeld, 1948; Campbell, 1954; Milbrath, 1981; Pizzorno, 1975; Conge, 1988; Pasquino, 1988; Sabucedo, 1989), también se atribuye a la formación universitaria un primer acercamiento con la construcción de ciudadanía y, por último, se exponen los resultados de la encuesta Latinobarómetro (2018) que contiene la percepción ciudadana sobre la democracia y la situación económica de los jóvenes, específicamente en los países donde hay elecciones en 2021. Lo anterior permite comprender el contexto actual en el cual se desarrollan las elecciones y las condiciones sociales que prevalecen en un sector importante de la población, los jóvenes. 


\section{Juventudes y democracia en América Latina}

A diferencia de Europa, Asia y África, donde los sistemas políticos se ubican entre democracias, dictaduras y sistemas socialistas, los regímenes políticos en América son predominantemente democracias contemporáneas, donde el sistema presidencialista está presente en casi todos los países americanos, a excepción de Canadá que formalmente es una monarquía constitucional, Haití, república semipresidencialista y Cuba, república socialista.

Los gobiernos democráticos en América Latina han mostrado preocupación por promover espacios de participación para los jóvenes. No obstante, los desafíos que los gobiernos enfrentan para construir un proyecto de nación, enfocado principalmente en fortalecer el desarrollo económico, demora la incorporación de la ciudadanía en la toma de decisiones, lo cual puede derivar en colapsos democráticos en un mediano plazo (Lührmann, 2019). Los jóvenes no permanecen inmóviles en el espacio público porque recrean vías renovadas de participación para ser escuchados y tomados en cuenta.

La estrategia de la juventud impulsada en Latinoamérica ha sido enmarcada en la agenda 2030, que define los Objetivos del Desarrollo Sostenible. Los estudios académicos sobre juventudes se han incrementado a partir de la década de los noventa, aunque estos no se traducen inmediatamente en políticas públicas por parte de los gobiernos. Sin embargo, dan cuenta de las acciones que instituciones como la UNES$\mathrm{CO}$ que, a través de la Oficina Regional de Educación para América Latina y el Caribe (OREALC/ UNESCO), monitorea los avances e impacto de las políticas y programas sociales en materia de educación (Rodríguez, 2010). De igual forma, el Organismo Internacional de Juventud para Iberoamérica (OIJ), se ha convertido en una institución de referencia en materia de juventud (OIJ, 2018) en la región.

En este contexto, los gobiernos de América Latina, mayoritariamente repúblicas presidencialistas, comparten diferencias sociales abismales, por tanto, la idea preconcebida de que la democracia logrará la igualdad social se ha desvanecido para dar paso a contribuciones recientes como la de Przeworski (2010), que permiten comprender los quiebres democráticos, debido a que la democracia siempre presentará desavenencias en su implementación por las desigualdades económicas de los ciudadanos, las cuales no se resolverán de manera inmediata, pero a pesar de ello, debe prevalecer el compromiso con la igualdad. De esta 
manera, para que la democracia llegue a tener éxito las fuerzas políticas deben asumir un compromiso racional y reflejarlo en las instituciones políticas con el fin de fortalecer la estabilidad del sistema democrático (Weingast, 1997).

Un sistema democrático no se piensa sin mecanismos que garanticen la participación ciudadana. Las vías institucionales varían considerablemente en cada nación porque influye la historia política que dio origen al sistema político vigente, además de la voluntad política de los gobiernos y los contextos globalizadores que inciden en las agendas nacionales.

Los jóvenes empujan fuertemente para que los gobiernos, sin importar el régimen político, reconozcan la capacidad de organización desde los espacios rurales, una característica especial de las naciones latinoamericanas, donde los jóvenes se desarrollan en contextos con mayor desigualdad social y menos oportunidades de desarrollo que el resto de la población.

En este contexto, los jóvenes están visibles en el centro de las grandes políticas de desarrollo social. A pesar de ello, las estrategias juveniles, aún conservan tareas pendientes que deben atenderse de manera prioritaria, como es la violencia, la discriminación, promover la igualdad de género y sociedades inclusivas, como parte de una estrategia para reducir la brecha de desigualdad social que cada vez es más visible; ciudadanos desiguales en un mundo donde se pugna por la igualdad donde la ruralidad parece alejada del centro mientras que las juventudes, a través de las tecnologías de comunicación, son capaces de reducir la dimensión espacial y colocarse en el centro de las decisiones para avanzar hacia una ciudadanía colectiva.

\section{Los jóvenes en América Latina}

La categoría juventud surge, en primer lugar, como un fenómeno urbano, de clases medias y altas (Souto, 2018), lo que dificultó identificar elementos propios de la actuación independiente de los jóvenes. Más tarde, la definición se orientó hacia las diferencias sociales entre el mundo urbano y rural, género, etnias, razas, escolaridad, lo que permitió una reconstrucción de símbolos que rodean al término juventud, que han ayudado a comprender las dinámicas juveniles, a través de los diversos 
abordajes teóricos que se circunscriben desde diferentes perspectivas para estudiar el mundo social y cultural que les rodea.

Hemos expresado en líneas anteriores que América Latina está inmersa en una serie de complejidades sociales, en el caso de los jóvenes destaca la población vulnerable como uno de los principales sectores sociales que requieren atención prioritaria. De acuerdo con estimaciones de la Comisión Económica para América Latina y el Caribe (CEPAL) alrededor del $20 \%$ de la población en América Latina reside en áreas rurales. En países como Argentina, Brasil, Chile, Uruguay y Venezuela, la población rural no alcanza el 20\%, mientras que los países de Centroamérica presentan población rural que supera el 40\% (Guiskin, 2019).

Las características demográficas de la población permiten identificar diferencias significativas entre la población urbana y rural. Por ejemplo, un estudio realizado en 2017 compara las pirámides de población entre ambas áreas, el resultado muestra un angostamiento de la base de la pirámide que indica una disminución de la natalidad. Sin embargo, la pirámide urbana muestra que la mayoría de la población se concentra en edades intermedias (Guiskin, 2019), lo que se traduce en un descenso de la natalidad y un mayor control de la mortalidad, mientras que la pirámide de la población rural indica una tendencia progresiva en la población mayormente joven y una menor proporción en adultos mayores. Lo anterior refleja que en áreas urbanas los jóvenes representan el grupo poblacional más numeroso y en las áreas rurales se observa una población menor.

Es evidente que, en las comunidades rurales, la emergencia juvenil esboza complejidades sociales como pobreza, exclusión, desempleo y condiciones precarias, que afectan a grupos de personas en condiciones de pobreza y exclusión, entre los que se encuentran los jóvenes. A pesar de que los estudios de la CEPAL (2018) reconocen un avance importante en la reducción de los niveles de pobreza y pobreza extrema, a partir del 2015 se observa un incremento relevante en estos índices, en gran medida se debe a la situación política y social que prevalece en Brasil y Venezuela.

Los jóvenes rurales son más vulnerables que los urbanos. En Colombia, de los jóvenes de 15 a 24 años que viven en zonas urbanas el $25,5 \%$ se encuentra en situación de pobreza, pero entre aquellos que viven en zonas rurales se encuentra en esta condición el 39,8\%. México registra el $39,7 \%$ de pobreza en jóvenes de 15 a 24 años que viven en zonas urbanas, porcentaje que se eleva al $41,3 \%$ entre aquellos radica- 
dos en zonas rurales. En Bolivia los jóvenes de zonas rurales concentran el 22,2\% en pobreza, mientras que en las zonas rurales este porcentaje se eleva al 48,9\%. En Paraguay, la pobreza en zonas urbanas registra 34, $8 \%$ de jóvenes, mientras que en las zonas rurales esta cifra se eleva al 51\% (Guiskin, 2019).

En este sentido, ser joven rural implica tener un lugar subordinado en la estructura social (Castelli, 2016). Las precariedades en las zonas rurales se acentúan más cuando se trata de pueblos indígenas, debido a que registran mayores niveles de pobreza y están más expuestos a la exclusión y discriminación social. La población indígena en países como Ecuador, Uruguay y Venezuela, reportan mayor proporción en áreas urbanas, mientras que en países como Brasil y México el porcentaje de población indígena es mayor en áreas rurales para el sector juvenil (Guiskin, 2019).

Antes de la pandemia la situación de vulnerabilidad social estaba muy arraigada en la región, sin embargo, como derivación de sus efectos económicos, las precariedades se han multiplicado, particularmente en temas relacionados con desempleo, pobreza, exclusión, acceso a la educación, entre otos. En 2021 hay elecciones presidenciales en Chile, Perú, Nicaragua y Honduras y elecciones intermedias en México y Argentina ¿qué condiciones económicas y políticas prevalecen en estos países para la población joven?

Entre 2006 y 2017 Chile redujo la pobreza del 19,6\% a 3,7\% y el porcentaje de población vulnerable disminuyó del 43,9\% al 30,1\% (Banco Mundial, 2021). Sin embargo, la desigualdad de ingresos ha provocado que la clase media en expansión perciba mayores desigualdades de oportunidades, porque la oferta de servicios de educación y atención de la salud se ha segmentado, situación que se ha agravado a raíz de la pandemia que ha impactado duramente en los sectores más vulnerables. La inestabilidad económica presente en el contexto político chileno demanda una inminente estrategia nacional para sumar consensos políticos que incentiven la recuperación económica.

La situación de Perú mostró uno de los crecimientos más acelerado de los países en América Latina entre 2002 y 2013, debido al fuerte crecimiento del empleo que impactó en la reducción de la pobreza; el crecimiento económico del 2014 a 2019 fue más lento, por lo que la inversión privada se redujo temporalmente, sin embargo, el manejo prudente de la política fiscal y monetaria han permitido al país atenuar las consecuencias económicas hasta antes de la pandemia, sin embargo, los 
efectos del COVID-19 han llevado a Perú a un descenso significativo del Producto Interno Bruto (PIB) del 17,4\% durante el primer semestre del 2020 (Banco Mundial, 2021), pero la oportuna actuación del gobierno a través de programas de compensación económica han permitido proteger a la población vulnerable.

En Nicaragua el crecimiento económico es incierto, a pesar de que en 2017 mantenía un buen ritmo. Los disturbios sociales y políticos que tuvieron lugar en 2018 provocaron un impacto negativo en la economía, aunado a los efectos de la pandemia. El Banco Mundial (2021) pronostica un declive en sectores como construcción, comercio y turismo, por lo que, lejos de reducirse la pobreza, podría incrementarse de manera significativa.

En los últimos años, Honduras había registrado las tasas de crecimiento económico más altas en Centroamérica, de acuerdo con el Banco Mundial (2021). A pesar de ello, el país enfrenta altos niveles de pobreza y desigualdad: un $48,3 \%$ de las personas vive en pobreza, porcentaje que se eleva en zonas rurales al 60,1\%. Adicionalmente, problemáticas como los altos niveles de violencia registrados en el 2018 clasifican a este país como uno de los que registran las tasas más altas de feminicidios en el mundo, a lo que debemos sumarle el decrecimiento económico debido a los efectos de la pandemia que impactará fuertemente en las actividades económicas del país.

En contraste, Argentina es una de las economías más sólidas de América Latina, con importante liderazgo en los sectores agrícolas y ganadero. No obstante, la pandemia ha agravado la situación de pobreza urbana, que en el 2020 alcanzó el 40,9\%, con un índice de indigencia el $10,5 \%$ y una pobreza infantil del 56,3\% (Banco Mundial, 2021). A pesar de las acciones del gobierno la pandemia impactó fuertemente en Argentina y se espera que se tenga un déficit fiscal mayor al 10\% del PIB.

Por último, México con una población de casi 130 millones de habitantes es una de las economías más grandes del mundo y la segunda de América Latina, a pesar de lo cual en las últimas tres décadas ha tenido un promedio de crecimiento estimado en poco más del 2,0\% anual, y a raíz de la pandemia la economía se contrajo un $8,2 \%$ en el año 2020 (Banco Mundial, 2021). Se espera que las políticas gubernamentales que se han centrado en apoyos económicos a la población vulnerable contribuyan en la recuperación económica. Para el 2021 se pronostica un crecimiento de la economía en 4,5\%. 
El comportamiento económico en estos países incidirá fuertemente en la toma de decisiones de los electores en relación con el voto. El decrecimiento de la economía se traduce en mayores niveles de pobreza en las familias, la población tomará la decisión de elegir a sus representantes, probablemente entre aquellos que estén orientados hacia una política económica distinta a la del gobierno en turno. Finalmente, la toma de decisiones de la ciudadanía también es influenciada por el poder adquisitivo. Los jóvenes, particularmente en los países con procesos electorales en puerta, muestran un distanciamiento mayor a los registrados en procesos anteriores debido a las consecuencias incalculables de la pandemia.

\section{Participación política y jóvenes en América Latina}

Los jóvenes están activos políticamente en todo el mundo, bajo diversas modalidades para hacerse visibles, en algunos casos, luchando contra el dominio del sistema político, como en el caso de la Primavera Árabe en 2010, en otros espacios conquistando jurídicamente derechos que garanticen condiciones idóneas para participar en política.

La participación política es tan antigua como el concepto mismo, sin embargo, los primeros trabajos publicados destacan Lazarsfeld, Berelson y Gaudet, (1948) y Campbell, Gurin y Miller (1954), quienes han aportado diferentes nociones para incorporar a la participación política como un tópico clásico de la ciencia política. El notable trabajo de Milbrath, publicado en 1965, enlista las formas posibles de comportamientos políticos cuyo propósito es incidir en las decisiones del gobierno. Con el paso de los años, estas modalidades de participación se han diversificado para dar paso a nuevas y modernas formas de organización. En 1975 Pizzorno concluyó que la participación política es una acción que se cumple en solidaridad con otras, en el ámbito de un Estado o de una clase con vistas a conservar o modificar la estructura de los sistemas dominantes, desarrollada dentro de las relaciones de poder. A fines de la década de los ochenta Conge (1988) aportó que la participación política puede ser: (a) verbal o escrita, (b) violenta o no violenta y (c) de distinta intensidad. A partir de esta clasificación, han surgido una serie de teorías que distinguen entre lo convencional y aquello que está al margen de la legalidad. 
En esa misma década, Pasquino (1988) definió a la participación política como aquel conjunto de actos y de actitudes dirigidos a influir de manera más o menos directa y más o menos legal sobre las decisiones de los detentadores del poder en el sistema político. En este mismo sentido, Sabucedo (1989) atribuye a la participación política las características de instrumental, voluntaria y no limitada por los criterios de legalidad. La participación política es activa e incluye conductas agresivas y no agresivas, así como conductas orientadas a mantener o cambiar la forma de gobierno, las autoridades gubernamentales y/o sus decisiones y no incluye acciones comunitarias ni las intenciones o consecuencias de las acciones. De tal forma que, la participación política tiene como propósito incidir en las distintas configuraciones del ámbito político, que involucra la toma de decisiones en las diferentes estructuras de gobierno.

De acuerdo con Juan Russo (2010), la participación política consiste en el compromiso que el individuo tiene con el sistema político, el cual se clasifica en dos niveles de actividad: la participación política visible y la participación latente. La primera se refiere al conjunto de acciones y comportamientos que apuntan a influir de modo más o menos directo y más o menos legal en las decisiones, mientras que, la segunda es potencial, pero en cierta medida efectiva, cuando hay una comunidad interesada en la política e informada sobre su dinámica, se activa raramente y en modo discontinuo, aunque con capacidad de participar.

Por lo anterior, debemos entender a la participación política como aquellas acciones voluntarias e invisibles, tanto individuales como colectivas, cuyo fin es incidir de manera directa y legal en las acciones públicas que los gobiernos llevan a cabo durante el tiempo que ejercen el poder. Por acciones invisibles entendemos aquellos comportamientos que no se activan políticamente por una razón de ser, debido a que existe un descontento sobre los actores políticos en el poder. Sin embargo, pueden activarse en cualquier momento e influir significativamente en las condiciones políticas de la población.

Precisamente, numerosos estudios a cargo de instituciones y organismos especialistas en temas de juventud, como la CEPAL, han señalado la lejanía y falta de confianza que expresan los jóvenes de América Latina respecto de la política, lo cual se traduce en una participación escasa en los procesos electorales (Maldonado, 2015). De esta manera, la juventud en América Latina enfrenta serios desafíos, cuyo origen se encuentra en la desigualdad social que ha permeado por varias décadas en esta región, donde los gobiernos han contribuido poco en promover 
la inclusión social y reducir la brecha que enfrentan los jóvenes para incorporarse socialmente en los temas públicos por la vía institucional.

La crisis sanitaria que se vive en el mundo entero, a partir del año 2020, ha permitido a los jóvenes involucrarse activamente desde acciones de prevención de la pandemia, hasta ofrecer apoyo ciudadano a través de grupos de solidaridad y altruismo. En cuanto al ámbito político, las redes sociales son los espacios virtuales preferidos por las juventudes donde es posible organizarse y provocar acciones de cambio.

Ante esta nueva configuración social que trae aparejada modernos esquemas de organización y participación ciudadana, diversas instituciones y organismos académicos han impulsado estudios recientes en América Latina, para analizar el impacto de la pandemia en las juventudes y un posible distanciamiento, aún mayor, en los asuntos públicos. Las estrategias recientes merecen analizarse y difundirse a través de los gobiernos nacionales para que sean incorporadas en las agendas públicas y lograr el anhelo democrático, la participación efectiva de los jóvenes.

\section{Expresiones juveniles desde las universidades públicas}

La construcción de las naciones democráticas, en América Latina, ha contado con el activismo de los jóvenes quienes, desde el terreno educativo, a través de acciones colectivas y no convencionales, han intentado combatir los gobiernos autoritarios o dictatoriales y abrir la puerta a la democracia electoral.

La década de los sesenta fue particularmente activa para las juventudes en América Latina; desde las universidades públicas la agitación estudiantil se hizo visible en varios países: Colombia en 1962 con la movilización estudiantil de la Universidad Nacional de Bogotá, movilizaciones que se extendieron hasta 1969 (Cruz, 2016) en Ecuador y Bolivia en 1964; Argentina en 1966, con la irrupción de la policía federal en la Universidad de Buenos Aires lo que fue llamada Noche de los Bastones Largos (Vera, 2013); en México en 1968, con la trágica matanza en Tlatelolco (Pozas, 2018) y Brasil en el mismo año, donde hubo fuertes represiones estudiantiles que ocasionó la muerte de un estudiante de secundaria, lo que provocó que miles de manifestantes se volcaran a las calles (Donoso, 2020).

Otros ejemplos recientes son el movimiento estudiantil del 2011 en Chile, como parte del rechazo al sistema educativo; en 2012 el movi- 
miento \#YoSoyl32 en México (Maldonado, 2015) y, recientemente, en 2019 en Colombia se generó una ola de protestas juveniles ante la inconformidad de pagar deudas de la nación con recursos de la educación.

Los movimientos estudiantiles son protestas contra el sistema autoritario y dictatorial de los gobiernos, cuyas luchas fueron inicialmente para defender la autonomía universitaria, posteriormente sirvieron de cimientos para edificar la democracia latinoamericana. La gran afluencia de jóvenes en esos eventos tuvo factores externos que la elevaron, como fue la muerte de Ernesto Guevara de la Serna (Che Guevara) en octubre de 1967. A partir de su muerte, la guerrilla cobró fuerza y la influencia del pensamiento político de la revolución cubana trascendió a varios países, entre ellos, México, donde el símbolo del Che Guevara ha sido el estandarte de innumerables marchas juveniles.

Los jóvenes que asisten a la universidad inician un proceso de reconocimiento como sujetos políticos y de derechos, es decir, reconocerse como ciudadanos, también es oportuno para acercarse a la política en cualquiera de sus formas y modalidades, convencional (mecanismos legales) o no institucional (la protesta y movilización colectiva). En contraparte, los jóvenes que no asisten a la escuela y asumen roles de adultos a temprana edad es posible que se alejen de los asuntos públicos.

En conclusión, la calidad de ciudadanía se inicia desde las aulas escolares, donde confluyen elementos culturales y sociales para generar escalas simbólicas en la realidad individual, adaptándose en pequeños espacios donde se forjan lazos con la comunidad política, posteriormente el aprendizaje empírico adquiere sentido cuando se exige frente al Estado.

\section{Estrategias en América Latina para promover la participación de los jóvenes durante la pandemia}

Desde que la pandemia causó estragos en las sociedades, la ciencia, en sus distintas disciplinas, empezó a realizar diferentes estudios para evaluar el impacto económico, político, social y cultural, analizando los efectos en los diferentes sectores sociales. De esta manera, grupos de investigación de la sociedad civil y académicos han realizado diferentes aportaciones para incentivar la participación de los jóvenes en los procesos democráticos que se desarrollan durante la pandemia en las naciones de América Latina. 
Así surge una iniciativa que promueve la Organización de las Naciones Unidas para el desarrollo sostenible en América Latina y el Caribe, que en mayo del 2020 emitió una encuesta para analizar cómo ha impactado la pandemia de COVID-19 en la juventud. Este instrumento tuvo la participación de cerca de 8000 jóvenes y los resultados se presentaron en un conversatorio virtual en el marco del Día Internacional de la Juventud el 13 de agosto del 2020. Los resultados preliminares señalan que 1 de cada 4 jóvenes se preocupa por su situación económica y casi 1 de cada 5 teme por su situación educacional; casi 1 de cada 3 personas jóvenes viven en comunidades donde hay escasez de alimentos y casi una de cada 6 no cuenta con los recursos para comprar alimentos básicos. Esta condición se eleva entre aquellos que viven en comunidades indígenas donde se reporta el 44, 9\% de personas jóvenes en esta condición (ONU, 2020).

En la encuesta también destacan temas relacionados con la educación: 4 de cada 10 jóvenes estudia de tiempo completo, condición que se da mayoritariamente entre adolescentes. Las personas jóvenes de origen afrodescendientes son las que menos se encuentran estudiando. En relación con el empleo de los jóvenes, casi 1 de cada 3 reporta que su entorno laboral se ha complicado debido a la pandemia, situación que se agrava en las personas con discapacidad; sobre este mismo rubro las mujeres jóvenes entre 20 a 24 años señalan que la carga de trabajo doméstico se ha incrementado en la pandemia. En relación con la participación de los jóvenes para combatir la pandemia casi 4 de cada 10 jóvenes señalan haber participado o liderado alguna acción en respuesta al COVID-19 (ONU, 2020). En este mismo sentido, la CEPAL ha realizado en el último año una serie de estudios para dar seguimiento a los efectos de la pandemia a través del Observatorio Demográfico y el Observatorio de Juventud para América Latina y el Caribe, atendiendo a las estadísticas de mortalidad y a los retos que enfrenta la educación durante el confinamiento (CEPAL, 2020).

Por otra parte, la Organización Internacional del Trabajo (OIT) realizó un estudio a nivel mundial a través de una encuesta dirigida a jóvenes de al menos 112 países del mundo, con el propósito de evaluar el impacto de la pandemia, particularmente las repercusiones en el empleo, la educación y el bienestar mental. Los hallazgos indican que aproximadamente el $17 \%$ de los jóvenes que trabajaban antes del inicio de la pandemia dejaron de hacerlo totalmente, disminuyeron las horas de trabajo, por lo que el $42 \%$ indicó una reducción en sus ingresos económi- 
cos. Es así como los jóvenes se encuentran más expuestos a la pobreza. El informe también destaca que las mujeres jóvenes indicaron pérdidas elevadas en cuanto a su productividad, en comparación con los hombres (OIT, 2020).

Los organismos internacionales han mostrado una gran preocupación por estudiar la influencia de la pandemia en los jóvenes, por lo que es indispensable que los gobiernos definan las estrategias para impulsar políticas y mecanismos que promuevan la reducción de las desigualdades sociales a las que jóvenes y adolescentes se enfrentan.

\section{Elecciones 2021 en América Latina}

De acuerdo con la información difundida por IDEA internacional (2020) todas las elecciones que estaban previstas en América Latina durante el segundo trimestre del año 2020 fueron pospuestas para segundo semestre del 2020, incluso una de ellas, Paraguay, fue postergada hasta el 2021. El único país que mantuvo su fecha original fue Venezuela. Estas decisiones de los países reflejan una serie de consensos políticos para que la legitimidad de los procesos no se vea afectada ante las reformas jurídicas que se tuvieron que realizar.

El desafío de las elecciones en el 2020, además de asegurar la salud de los electores, tuvo un componente político significativo: innovar los procesos electorales al mismo tiempo que se debía garantizar el mayor grado de confianza, legitimidad y credibilidad en el proceso, para alcanzar el nivel más alto de participación electoral. El reto fue evitar que la democracia fuera una víctima más de la pandemia.

De esta forma, se aceleró la incorporación de soluciones digitales. Aunque no derivaron en el voto electrónico porque este requiere de una serie de ajustes jurídicos, tecnológicos y de capacitación que deben ser valorados para las elecciones del 2021, la innovación de las campañas virtuales es un tema que debe analizarse con mucho esmero. Las redes sociales fueron empleadas como principales espacios de difusión de contenido político, pero quizá cabe indagar si es suficiente para el elector la información que recibe de manera virtual y ¿qué pasa con aquellas personas que no tienen acceso a redes sociales?

Ahora bien, la participación electoral tuvo un comportamiento diferente en cada país, en el caso de Bolivia y Uruguay registró una participación por encima del 85\%. En República Dominicana hubo una dis- 
minución de 14 puntos. En las municipales de Brasil también hubo una disminución en la intención del voto y un revés a los candidatos apoyados por el presidente actual. Venezuela fue el país que mayor nivel de abstención tuvo: 70\% (IDEA Internacional, 2020).

En 2021 las elecciones presidenciales en Chile, Perú, Nicaragua y Honduras, así como las elecciones intermedias que se celebrarán en Argentina y México, en un contexto de pandemia, representan un verdadero desafío, pero con experiencias previas del 2020, tanto para las instituciones encargadas de organizar los procesos electorales, como para la ciudadanía y los actores políticos involucrados frente a los cambios derivados de la nueva configuración política y la incorporación de nuevos esquemas electorales.

Durante la pandemia los jóvenes han mostrado interés en actividades de voluntariado para apoyar a las poblaciones con mayores carencias de recursos económicos (OIT, 2020), pero ¿qué hay de la política? Es fundamental que los jóvenes formen parte de los procesos electorales debido a la experiencia y sensibilidad que han adquirido durante la pandemia, el gran interrogante aquí es si los jóvenes se involucrarán activamente en los procesos electorales como en las actividades de apoyo social.

En 2018 la Corporación Latinobarómetro, con sede en Santiago de Chile, publicó los resultados de la encuesta más reciente que desde 1995 realiza en 18 países de América Latina, la cual aplicó metodología estadísticamente representativa (Latinobarómetro, 2018). Para este trabajo se tomaron como referencia seis respuestas, de la base de datos, que dieron las personas jóvenes, entre el rango de 18 a 29 años, en los seis países donde hay elecciones en 2021, (véase tabla 1). 


\section{Tabla 1: Opinión de personas jóvenes (18 a 29 años)}

\begin{tabular}{|c|c|c|c|c|c|c|c|}
\hline Preguntas & Respuestas & Argentina & Chile & Honduras & México & Nicaragua & Perú \\
\hline Imagen del país & $\begin{array}{c}\text { Está } \\
\text { progresando }\end{array}$ & 9.7 & 31.8 & 23.0 & 14.0 & 9.4 & 23.1 \\
\hline & Está estancado & 49.0 & 47.5 & 49.7 & 67.7 & 51.4 & 52.7 \\
\hline & Está en & 41.3 & 20.7 & 27.2 & 18.3 & 39.2 & 24.1 \\
\hline & Total & 100.0 & 100.0 & 100.0 & 100.0 & 100.0 & 100.0 \\
\hline Satisfacción con el & Muy satisfecho & 2.4 & 1.7 & 7.9 & 2.6 & 7.5 & 2.5 \\
\hline $\begin{array}{l}\text { funcionamiento } \\
\text { de la economía en }\end{array}$ & $\begin{array}{l}\text { Más bien } \\
\text { satisfecho }\end{array}$ & 5.6 & 30.8 & 9.2 & 12.0 & 10.2 & 8.4 \\
\hline & No muy & 48.3 & 35.7 & 46.5 & 55.7 & 38.7 & 52.0 \\
\hline & Nada satisfecho & 438 & 318 & 365 & 296 & 436 & 371 \\
\hline & Total & 100.0 & 100.0 & 100.0 & 100.0 & 100.0 & 100.0 \\
\hline Grado de & Muy satisfecho & 5.2 & 4.2 & 12.1 & 2.6 & 8.2 & 4.8 \\
\hline $\begin{array}{l}\text { satisfacción con el } \\
\text { funcionamiento }\end{array}$ & $\begin{array}{l}\text { Mas bien } \\
\text { satisfecho }\end{array}$ & 22.7 & 41.1 & 14.5 & 15.4 & 11.3 & 5.0 \\
\hline de la democracia & $\begin{array}{c}\text { No muy } \\
\text { satisfecho }\end{array}$ & 47.9 & 39.6 & 46.3 & 51.2 & 37.5 & 63.0 \\
\hline & Nada satisfecho & 24.1 & 15.1 & 27.1 & 30.8 & 43.1 & 27.2 \\
\hline & Total & 100.0 & 100.0 & 100.0 & 100.0 & 100.0 & 100.0 \\
\hline Confianza en los & Mucha & 1.7 & 0.0 & 3.4 & 1.5 & 4.2 & 0.5 \\
\hline partidos políticos & Algo & 16.0 & 13.4 & 7.7 & 10.8 & 8.3 & 7.1 \\
\hline & Poca & 36.8 & 31.0 & 30.2 & 35.0 & 22.2 & 39.2 \\
\hline & Ninguna & 45.5 & 55.6 & 58.8 & 52.8 & 65.3 & 53.2 \\
\hline & Total & 100.0 & 100.0 & 100.0 & 100.0 & 100.0 & 100.0 \\
\hline $\begin{array}{c}\text { ¿Cómo diría Ud. } \\
\text { que es la }\end{array}$ & $\begin{array}{c}\text { Una democracia } \\
\text { plena }\end{array}$ & 2.4 & 4.8 & 3.4 & 1.7 & 7.4 & 3.5 \\
\hline democracia en su & Una democracia & 30.6 & 53.8 & 18.8 & 27.0 & 15.4 & 21.5 \\
\hline & problemas & & & & & & \\
\hline & $\begin{array}{c}\text { Una democracia } \\
\text { con grandes } \\
\text { problemas }\end{array}$ & 50.5 & 34.4 & 44.4 & 49.0 & 30.3 & 51.5 \\
\hline & $\begin{array}{l}\text { No es una } \\
\text { democracia }\end{array}$ & 5.2 & 5.5 & 21.7 & 12.2 & 38.0 & 11.9 \\
\hline & $\begin{array}{l}\text { No entiendo lo } \\
\text { que es una } \\
\text { democracia }\end{array}$ & 11.3 & 1.5 & 11.7 & 10.1 & 8.9 & 11.6 \\
\hline & Total & 100.0 & 100.0 & 100.0 & 100.0 & 100.0 & 100.0 \\
\hline Aprobación de la & Aprueba & 23.3 & 40.2 & 35.2 & 14.0 & 24.6 & 26.7 \\
\hline gestión del & No aprueba & 76.7 & 59.8 & 64.8 & 86.0 & 75.4 & 73.3 \\
\hline $\begin{array}{l}\text { gobierno que } \\
\text { encabeza el } \\
\text { presidente }\end{array}$ & Total & 100.0 & 100.0 & 100.0 & 100.0 & 100.0 & 100.0 \\
\hline
\end{tabular}

Fuente: elaboración propia a partir de la base de datos de Latinobarómetro (2018) 
Los resultados muestran una importante relación entre la percepción del desarrollo del país con el grado de satisfacción de la democracia. Es decir, las condiciones económicas tienen un efecto significativo en la opinión de las personas jóvenes respecto de los gobiernos. La mayoría no está satisfecha con el funcionamiento de la economía en su país, a excepción de Chile que, de los seis países analizados, muestra mayor satisfacción por las condiciones económicas que prevalecen en su país.

La desconfianza hacia los partidos políticos es una constante en todos los países analizados. Sin embargo, llama la atención que en Nicaragua el $65,3 \%$ no tiene ninguna confianza, por lo que también registra el mayor grado de insatisfacción con la democracia, con el 43,1\%, resultado de la enorme crisis sociopolítica del país. El modelo económico y político impulsado por Daniel Ortega, enfocado en el nacionalismo revolucionario, es un modelo que ha agotado sus expectativas, por lo que la población joven exige más libertades y mayor participación en la toma de decisiones.

La aprobación que los jóvenes realizan respecto de sus gobiernos, antes de que iniciara la pandemia, era escasa. Únicamente Chile registra un asentimiento del $40,2 \%$. Es probable que después de la pandemia la percepción ciudadana sea muy diferente, más severa y crítica hacia las acciones del gobierno para evitar la propagación de la pandemia. Se esperaría que toda la experiencia acumulada durante la contención de los contagios sea canalizada en expresiones ciudadanas. No obstante, ante la ausencia de espacios colectivos que incentiven e involucren a la ciudadanía en la toma decisiones, los bajos índices de participación ciudadana seguirán manifestándose como uno de los tantos efectos que ha dejado la pandemia.

\section{Reflexiones finales}

Las experiencias electorales que se tuvieron en 2020 son una oportunidad de mejora para las elecciones del 2021. Si bien cada país tiene particularidades específicas, el propósito es asegurar el mayor número de electores y que ningún sector quede excluido, en este caso, lo que nos ocupa son los jóvenes. Sin embargo, el desgaste económico es mayor este año, tanto para el electorado como el gobierno y las instituciones electorales que disponen de poco tiempo y escasos recursos económicos para organizar la logística electoral, procurar la salud de los electores, 
garantizar confianza y legitimidad en el proceso y asegurar que todos los sectores sociales de manera equitativa acudan a emitir su voto. La distancia social no debe ser un impedimento para participar en las elecciones y los jóvenes no deben ser excluidos en la toma de decisiones.

La pandemia que provocó el nuevo coronavirus ha tenido un mayor impacto en América, principalmente en aquellas naciones con índices de pobreza superiores, acelerando el impacto en las juventudes en todos los ámbitos de su existencia (OIT, 2020). De esta manera, los vínculos sociales y políticos se verán inevitablemente afectados por la fragilidad emocional en la que se encuentran, además de las precarias condiciones económicas que predominan en la población, con mayor dureza en las zonas rurales. Aunado a ello, la baja confianza hacia las instituciones electorales y las modificaciones de las estrategias de promoción del voto serán claves para entender la escasa participación electoral de los jóvenes, pero probablemente la participación política encuentre nuevos caminos en las estrategias digitales que emplean los partidos políticos para promover las campañas electorales.

La relación entre el binomio política y juventud esboza un importante abordaje hacia los contrastes y devenires que dan vida a diversas formas de participación política, que los jóvenes emprenden como parte de las emergencias propias de los contextos socioculturales que prevalecen en la realidad que les envuelve. Los esquemas de participación política que adoptan los jóvenes permiten visibilizar las enormes desigualdades que les rodean. La no participación también enmarca realidades de subordinación o hartazgo de las estrategias y acciones de los gobiernos. Por lo tanto, las experiencias de participación que los jóvenes asumen, desde la individualidad, exponen una serie de simbolismos que describen la realidad que les rodea. Aún en el silencio, son capaces de comunicar el panorama social que impide un desarrollo pleno.

\section{Referencias bibliográficas}

Allin, S., Haldane, V., Jamieson, M., Marchildon, G., Morales Vázquez, M., y Roerig. (2020). Comparación de politicas de respuesta al COVID-19 en paises de la Región de América Latina y el Caribe. Washington, Estados Unidos: Banco Mundial.

Banco Mundial. (2021). Paises y economias. Recuperado el 10 de marzo de 2021, de https://datos.bancomundial.org/pais 
Castelli, L. (2016). Producción de desigualdades entre jóvenes rurales: relaciones intergeneracionales, normativa y desarrollo agroindustrial. En Castelli, L. Juventud y desigualdades en América Latina y el Caribe, (pp. 49-70). Buenos Aires, Argentina: CLACSO.

CEPAL. (2018). Panorama social de América Latina 2017. Santiago, Chile: CEPAL.

CEPAL. (2020). El impacto del COVID-19 en América Latina y el Caribe. Santiago, Chile: CEPAL.

CEPAL. (2020). La educación en tiempos de la pandemia de COVID-19. Santiago, Chile: CEPAL/UNESCO.

CEPAL. (2021). Observatorio Demográfico, 2020. Mortalidad por COVID-19 Evidencias y escenarios. Santiago, Chile: Naciones Unidas/ CEPAL.

Cruz, E. (2016). La izquierda se toma la universidad. La protesta universitaria en Colombia durante los años sesenta. Izquierdas, (29), 205-232.

Donoso, R. A. (2020). Movimientos estudiantiles de Brasil y México en 1968: análisis comparativo de sus demandas. Historia y memoria, 269-298.

Guiskin, M. (2019). Situación de las juventudes murales en América Latina y el Caribe. México, México: CEPAL/FIDA.

IDEA Internacional. (2020). Elecciones $y$ «COVID-19»: Elecciones de América Latina. Disponible en: http://bcn.cl/2expy.

Latinobarómetro. (2018). Informe 2018. Santiago, Chile: Latinobarómetro.

Lührmann, A. y. (2019). A Third Wave of Autocratization is Here: What is New About It? Democratización, 26, 7.

Maldonado, C. F. (2015). Participación política, apego a la democracia y temas prioritarios de las personas jóvenes en América Latina, 20002013. En Trucco, D. y Ullmann, H. Juventud: realidades y retos para un desarrollo con igualdad, (pp. 207-259). Santiago, Chile: CEPAL.

Milbrath, L. W. (1981). Polítical Participation. En Long, S. L. The handbook of political behavior, (pp197-237). Nueva York, Estados Unidos: Springer.

Organismo Internacional de la Juventud (OIJ) (2018). Libro blanco de 
politicas públicas de juventud. Madrid, España: OIJ/INAP/Pacto Juventud y Cooperación Española.

Organización Internacional del Trabajo (OIT) (2020). Los jóvenes y la COVID-19. Suiza: OIT.

Organización de las Naciones Unidas (ONU) (2020). Jóvenes ante Covid-19 en América Latina y el Caribe. Presentación preliminar de resultados. América Latina: ONU.

Pasquino, G. (1988). Mamual de ciencia política. Madrid, España: Alian$\mathrm{za}$.

Pizzorno, A. (1975). Participación y cambio social en la problemática contemporánea. Buenos Aires, Argentina: Siap Planteos.

Pozas, R. (2018). Los años sesenta en México: la gestación del movimiento social de 1968. Revista Mexicana de Ciencias Politicas y Sociales, (234), 111-132.

Przeworski, A. (2010). Qué esperar de la democracia: limites y posibilidades del autogobierno. Buenos Aires, Argentina: Siglo XXI.

Rodríguez, E. (2010). Politicas Públicas de Juventud en América Latina. Brasil: UNESCO.

Russo, J. (2010). Calidad democrática, formación ciudadana y comportamiento electoral. México, México: IFE/CONACyT.

Souto Kustrín, S. y Lucci, M. (2018). Historia de los Jóvenes de América Latina. Revista Paginas, 10 (22), 3-15.

Vera de Flachs, M. C. (2013). Universidad, dictadura y movimientos estudiantiles en Argentina. Córdoba 1966-1974. Revista Historia de la Educación Latinoamericana, 15 (21), 191-228.

Weingast, B. (1997). The political foundations of democracy and the rule of the law. American political science review, 91(2), 245-263. 